\title{
Management of people with epilepsy during the COVID-19 pandemic: a national survey among epileptologists in China
}

\author{
Zhenxu Xiao ${ }^{1}$, Ding Ding ${ }^{1}$, Shichuo $\mathrm{Li}^{2}$ and Zhen Hong ${ }^{1,2^{*}}$
}

\begin{abstract}
Background: Compared to the healthy people, people with comorbid medical conditions are more vulnerable in the context of the coronavirus disease 2019 (COVID-19) pandemic, including the people with epilepsy. Besides a consensus recommendation by multi-national epilepsy specialists, the situation of the epilepsy management during the pandemic has seldom been reported.

Methods: The China Association Against Epilepsy carried out an online nationwide survey among its board members in April 2020. One hundred and thirty board members from 22 provinces, 5 autonomous regions, and 4 municipalities across China responded to the questionnaires. They reported the situation of clinical practice and gave opinions on the management of people with epilepsy between January 13th and March 31st, 2020, a time period concentrated with confirmed COVID-19 cases.

Results: The proportions of patients consulting through telephone or online (88.4\%) and of patients with regular case review (93.9\%) were highest in the high-risk area, as reported by the responders. The patients in the high-risk area were more likely to have increased episodes of seizures (17.7\%), aggravated psychological disorders (30.2\%), and less accessibility to anti-seizure medications (ASMs) (77.2\%). Regular ASMs supply (74.6\%), medical consultation (69.2\%), and psychological aids (29.2\%) were urgently needed for people with epilepsy.

Conclusions: This study demonstrated the most common dilemma faced by people with epilepsy in policy circumstances during the COVID-19 epidemic in China. The opinions raised by Chinese epileptologists may provide reference for epilepsy care in other countries.
\end{abstract}

Keywords: COVID-19, Epilepsy, Survey, Consensus, Opinion, China

\section{Background}

On March 12, 2020, the coronavirus disease 2019 (COVID-19) was declared as a pandemic by the World Health Organization (WHO) [1]. As a response to the COVID-19 outbreak, China implemented strict quarantine measures in most communities and villages, and imposed a city lockdown in Wuhan. Compared to the healthy people, people with comorbid medical conditions are more vulnerable in the context of this public

\footnotetext{
* Correspondence: profzhong@sina.com

${ }^{1}$ Institute of Neurology, Huashan Hospital, Fudan University, Shanghai 200040, China

${ }^{2}$ China Association Against Epilepsy, Beijing, China
}

health issue, including the people with epilepsy. A consensus recommendation by multi-national epilepsy specialists suggests that people with epilepsy receive medical care at home rather than in health care facilities, and take measures to reduce the possibility of seizure exacerbation by adhering to the medications [2]. The impact of the COVID-19 pandemic on people with epilepsy in Italy [3] and Arabia [4] has been reported recently. A Chinese study has revealed severe psychological distress among people with epilepsy during this crisis [5]. However, on the other aspect, the management of people with epilepsy during the pandemic in China has not been reported.

(c) The Author(s). 2020 Open Access This article is licensed under a Creative Commons Attribution 4.0 International License, which permits use, sharing, adaptation, distribution and reproduction in any medium or format, as long as you give appropriate credit to the original author(s) and the source, provide a link to the Creative Commons licence, and indicate if changes were made. The images or other third party material in this article are included in the article's Creative Commons licence, unless indicated otherwise in a credit line to the material. If material is not included in the article's Creative Commons licence and your intended use is not permitted by statutory regulation or exceeds the permitted use, you will need to obtain permission directly from the copyright holder. To view a copy of this licence, visit http://creativecommons.org/licenses/by/4.0/. 
In April 2020, the China Association Against Epilepsy (CAAE) conducted a nationwide retrospective survey among epileptologists, aiming to assess the situation of management of people with epilepsy during the COVID-19 epidemic in China. In this article, we report the results of the survey and comment on the major problems and challenges of epilepsy management during the COVID-19 epidemic from the perspective of Chinese epileptologists.

\section{Materials and methods}

\section{Survey participants}

The CAAE is a national non-government organization dedicated to the treatment, prevention, and control of epilepsy in China. It is a non-profit association of neurologists, neurosurgeons, pediatric neurologists, and other experts serving epilepsy care nationwide. All board members of the CAAE are epileptologists with a MD degree from second-grade class-A or third-grade class-A hospitals. Two hundred and thirty-five board members of the CAAE were eligible survey participants.

\section{Questionnaire}

On April 3, 2020, the CAAE sent a two-part questionnaire to all the board members by email. The first part of the questionnaire quantitatively assessed the clinical practice situation in case management of people with epilepsy between January 13 and March 31, 2020, when the number of confirmed COVID-19 cases rose from 41 to 81554 in China $[6,7]$. The survey questions to each board member included: (1) the number of patients for consultation (including clinical consultation and other forms of teleconsultation); (2) the proportion of telephone/internet-based consultation; (3) the proportions of new presentations and regular case reviews (follow-ups) in all patients (including face-to-face clinic patients and telemedical patients); (4) the proportions of patients with increased episodes of seizures, with status epilepticus, and with aggravated psychological disorders, among the regular case reviews; (5) the proportion of patients who should but did not come for regular case review; and (6) the proportion of patients who were unable to obtain anti-seizure medications (ASMs). In the second part, 5 questions were designed to qualitatively elicit opinions from each board member: (1) What are the causes for seizure aggravation or increased frequency of seizure in patients for case review? (2) What are the reasons for the aggravation of psychological disorders (e.g. anxiety or depression) in patients for case review? (3) Why didn't the patients come for regular case review albeit they should? (4) Why were the patients unable to obtain ASMs? (5) What are the urgent needs for people with epilepsy in this COVID-19 epidemic?

\section{Statistical analysis}

Three groups of geographic area were defined according to the accumulating number of confirmed COVID-19 cases by April 20, 2020: the high-risk area identified for Hubei Province due to the highest number of cases, the low-risk areas identified for 15 provinces with $<300$ confirmed cases, and the moderate-risk areas for the other 15 provinces. The continuous variables are presented as median and range, while the categorical variables are presented as number and frequency (\%). Continuous variables with abnormal distribution were analyzed with the Kruskal-Wallis test, and the categorical variables were analyzed with the Pearson chi-square test and Fisher's exact test when applicable.

\section{Results}

As the high-risk area, Hubei Province had the largest number of COVID-19 cases $(n=68100)$, and the highest gross domestic product (GDP) compared with the lowand moderate-risk areas. However, there was no significant difference in the proportion of the urban population, the number of beds and practitioners, and the epilepsy prevalence among the three levels of epidemic areas (Table A.1).

One hundred and thirty CAAE board members from 22 provinces, 5 autonomous regions, and 4 municipalities completed the questionnaires. Among them, 3 were from the high-risk area, 89 were from the moderate-risk areas, and 38 were from the low-risk areas. Table 1 showed the characteristics of the people with epilepsy reported by the CAAE board members during the survey. The high-risk area had the highest proportions of patients consulting through telephone or online manners (88.4\%) and of patients with regular case review (93.9\%). Among the patients with case review, those in the highrisk area were more likely to have increased episodes of seizures (17.7\%) and aggravated psychological disorders (30.2\%), such as anxiety and depression. In addition, $60.2 \%$ of the patients in the high-risk area should but did not come for regular case review. A higher proportion of patients $(77.2 \%)$ in the high-risk area was unable to obtain ASMs, compared to those in low- and moderate-risk areas.

Table 2 shows the three most common answers for each subjective question. According to the answers of 130 responders, withdrawal, decreased dosage, alternative ASMs, and ASMs shortage were among the leading causes of aggravation or increased frequency of seizure (81.5\%), while lifestyle changes (26.9\%) and psychological disorders, such as anxiety and fear (18.5\%) were also reasons for poor disease management. The major causes for the aggravation of psychological disorders were ASMs shortage (54.6\%), fear of suffering from COVID-19 (40.8\%), and irregular lifestyle (14.6\%). For patients who 
Table 1 Characteristics of people with epilepsy reported by the CAAE board members during the survey

\begin{tabular}{|c|c|c|c|c|c|}
\hline \multirow[t]{2}{*}{ Variates } & \multirow[t]{2}{*}{ All } & \multicolumn{3}{|c|}{ COVID-19 epidemic severity areas } & \multirow[b]{2}{*}{$P$ value } \\
\hline & & Low-risk area & Moderate-risk area & High-risk area & \\
\hline Number of surveyed responders, $n$ & 130 & 38 & 89 & 3 & \\
\hline $\begin{array}{l}\text { Average number of patients with consultation reported per } \\
\text { responder, median (range) }\end{array}$ & $155(6,1400)$ & $115(18,1400)$ & $179(6,1210)$ & $200(150,766)$ & \\
\hline Telephone- or internet-based consultation ${ }^{\mathrm{a}}, n(\%)$ & $81(32.3)$ & $93(38.8)$ & $67(26.9)$ & $329(88.4)$ & $<0.001$ \\
\hline Patients with regular case review ${ }^{a}, n(\%)$ & $172(68.6)$ & $159(66.5)$ & $171(68.2)$ & $349(93.9)$ & $<0.001$ \\
\hline Patients with increased episodes of seizures ${ }^{\mathrm{b}}, n(\%)$ & $26(15.4)$ & $26(16.3)$ & $25(14.9)$ & $62(17.7)$ & 0.006 \\
\hline Patients with status epilepticus ${ }^{\mathrm{b}}, n(\%)$ & $2(0.9)$ & $1(0.8)$ & $2(1.0)$ & $3(0.9)$ & 0.184 \\
\hline Patients with aggravation of psychological disorders ${ }^{\mathrm{b}}, n(\%)$ & $32(18.8)$ & $42(26.2)$ & $26(15.1)$ & $106(30.2)$ & $<0.001$ \\
\hline $\begin{array}{l}\text { Patients who did not come for regular case review albeit } \\
\text { they should }{ }^{c}, n(\%)\end{array}$ & $460(73.3)$ & $724(82.0)$ & $345(67.6)$ & $527(60.2)$ & $<0.001$ \\
\hline Patients who were unable to obtain ASMs $^{\mathrm{a}}, n(\%)$ & $88(35.0)$ & $94(39.4)$ & $78(31.1)$ & $287(77.2)$ & $<0.001$ \\
\hline
\end{tabular}

ASMs antiseizure medications

${ }^{\mathrm{a}} \mathrm{Among}$ all the patients with consultation; ${ }^{\mathrm{b}} \mathrm{Among}$ patients with regular case review; ${ }^{\mathrm{c} A m o n g}$ all patients who should come for regular case review; $n$ (\%), the average number of patients reported per responder (\%)

should but did not come for regular case review, quarantine and traffic restrictions (73.8\%), fear of suffering from COVID-19 (33.8\%), and the epidemic of COVID19 (15.4\%) were major obstacles. Breakdown of ASMs supply systems (51.5\%), quarantine and traffic restrictions $(47.7 \%)$, and suspension of clinical services $(6.9 \%)$ were the main causes of ASMs inaccessibility. As suggested by the responders, maintaining regular ASMs supply (74.6\%), providing consultation, especially ASMs treatment instruction (69.2\%), and psychological aids (29.2\%) were the most urgent needs for people with epilepsy in the COVID-19 epidemic.

Table 2 The top three answers for each qualitative question

\begin{tabular}{|c|c|}
\hline Items & $\begin{array}{l}\text { Responders } \\
(N=130)\end{array}$ \\
\hline \multicolumn{2}{|c|}{ What are the causes of seizure aggravation or increased frequency in patients for case review? } \\
\hline Withdrawal, decreased dosage, alternative ASMs, and ASMs shortage, $n(\%)$ & $106(81.5)$ \\
\hline Lifestyle change, $n(\%)$ & $35(26.9)$ \\
\hline Psychological disorders (anxiety and fear), $n$ (\%) & $24(18.5)$ \\
\hline \multicolumn{2}{|c|}{$\begin{array}{l}\text { What are the reasons for the aggravation of psychological disorders (e.g. anxiety or depression) } \\
\text { in patients for case review? }\end{array}$} \\
\hline ASMs unavailability; ASMs shortage, $n(\%)$ & $71(54.6)$ \\
\hline Fear of suffering from COVID-19, $n(\%)$ & $53(40.8)$ \\
\hline Irregular lifestyle, $n(\%)$ & 19 (14.6) \\
\hline \multicolumn{2}{|l|}{ Why didn't the patients come for regular case review albeit they should? } \\
\hline Quarantine and the traffic restrictions, $n(\%)$ & $96(73.8)$ \\
\hline Fear of suffering from COVID-19, $n$ (\%) & $44(33.8)$ \\
\hline Epidemic of COVID-19, n (\%) & $20(15.4)$ \\
\hline \multicolumn{2}{|l|}{ Why were the patients unable to obtain ASMs? } \\
\hline Breakdown of ASMs supply systems, $n$ (\%) & $67(51.5)$ \\
\hline Quarantine and traffic restrictions, $n(\%)$ & $62(47.7)$ \\
\hline Suspension and limitations of clinical services, $n(\%)$ & $9(6.9)$ \\
\hline \multicolumn{2}{|l|}{ What are the urgent needs for people with epilepsy in this COVID-19 crisis? } \\
\hline Maintaining regular ASMs supply, $n$ (\%) & $97(74.6)$ \\
\hline Providing consultation, especially ASMs treatment instruction, $n(\%)$ & $90(69.2)$ \\
\hline Psychological counsultation, $n(\%)$ & $38(29.2)$ \\
\hline
\end{tabular}




\section{Discussion}

In this nationwide survey, we found that people with epilepsy from the low-, moderate- and high-risk areas had significant differences in approach to seeking medical consultation, psychological conditions, and ASMs accessibility. Regular ASMs supply, medical consultation, and psychological aids were the most urgent demands of them in the COVID-19 epidemic in China. To our knowledge, this is one of the few expert consensuses focusing on people with epilepsy during the COVID-19 pandemic.

The consensus on the impacting factors for epilepsy care during the COVID-19 epidemic (Fig. 1) may explain the poor management of epilepsy in China, reflected by the finding that patients in the high-risk area were more likely to have increased episodes of seizure and aggravation of psychological disorders (Table 1). According to the views of the epileptologists, the availability of ASMs seems to have played a crucial role in epilepsy management. Withdrawal or dose change would impact the efficacy of ASMs and cause the failure of seizure control. On the other hand, ASMs shortage would cause stress and anxiety for people with epilepsy, which may potentially cause aggravation of seizure. Therefore, the medication delivery systems should be optimized and maintained, and doctors should provide a long-time prescription of ASMs for convenience. A recent-published multi-national expert consensus also emphasized the importance of ASMs adherence and supply during the COVID-19 pandemic [2]. The percentages of patients with worsening of seizures/increased seizure frequency were $18 \%$ and $29.5 \%$ in Italy and Saudi Arabia, respectively $[3,4]$, which were higher than our result. Since these studies had different data collection methods, the discrepancy should be interpreted with caution.
Quite a few people with epilepsy need medical consultation during the lockdown period, especially for those who had increased episodes of seizure or status epilepticus. The difficulty to obtain professional medical advice from epileptologists would also arouse the anxious emotion, which could cause worse disease management. Under the nationwide strict lockdown and quarantine measures in China, telemedicine played an important role in delivering medical services for people with epilepsy. Online or telephone-based consultations could avoid person-to-person contact, and were extremely convenient and helpful in the high-risk area [2]. Therefore, we recommend people with epilepsy take full advantage of the telemedicine resources.

Psychological disorders, including anxiety, depression, panic, and stress, are common among people with epilepsy (18.8\%). Many modifiable and unmodifiable factors can contribute to the mental health issue. A study in southwest China has concluded that the psychological distress is highly prevalent among people with epilepsy (13\%) during the COVID-19 epidemic in China [5]. However, in a questionnaire-based research project in Saudi Arabia, $59.4 \%$ of people with epilepsy reported increased stress [4]. The different definitions of psychological stress in these studies may explain the diverse results.

An unhealthy lifestyle is another factor for aggravation of seizure, e.g. being addicted to electronic products and television, alcoholism, lacking physical exercises, and an irregular diet. Thus, psychological aids should also include the education of a healthy lifestyle for people with epilepsy.

This survey investigated the situation of people with epilepsy between January and March, the earlyto-mid stage of the epidemic in China. Through the

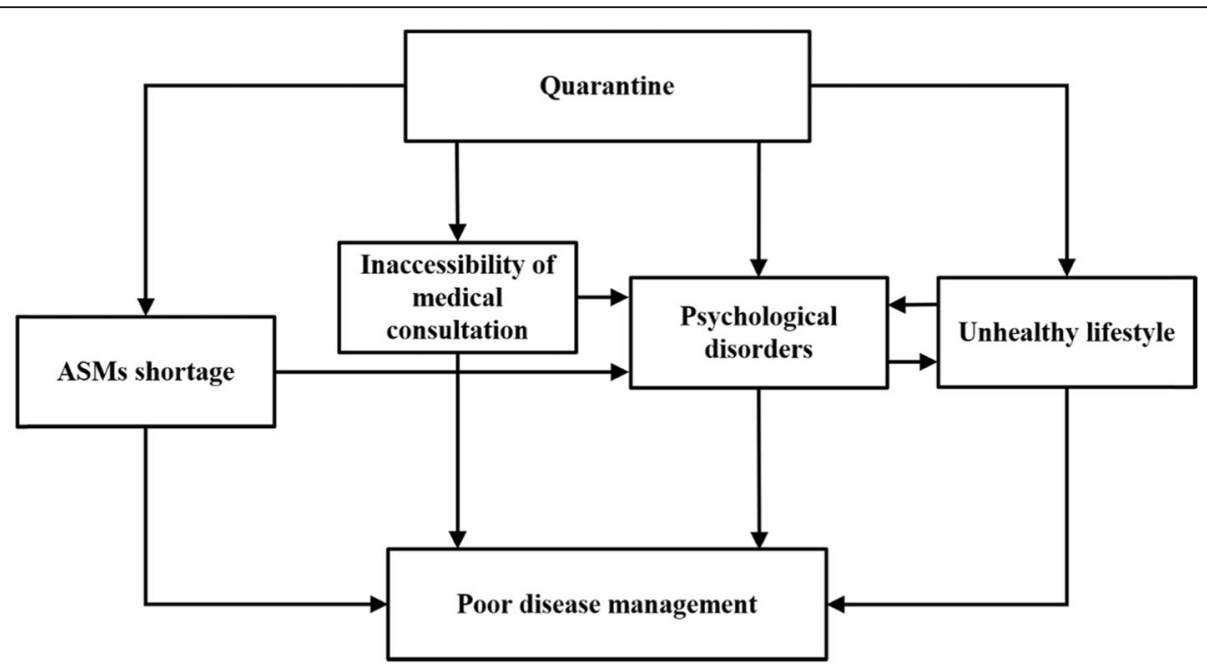

Fig. 1 Summary of the most-frequently suggested impacting factors on epilepsy care during the COVID-19 pandemic. ASMs, antiseizure medications 
efforts of the whole nation, China has already come through the severe situation. However, many countries are still experiencing the hardest time. The results of the survey may provide reference with respect to the management of people with epilepsy during the peak stage of the COVID-19 pandemic. Empirically, the epileptologists were likely to be more skillful to tackle problems during the later stage of the epidemic. Future studies need to focus on the evaluation of strategies by epileptologists in the later period of this global crisis.

The current study has several limitations. First, the response rate in the high-risk and moderate-risk areas are $100 \%$ and $98 \%$. Only $38 \%$ of board members responded to the survey in low-risk areas, and this may cause a selection bias. Second, our survey contained subjective answers only based on the responders' estimation. For example, the psychological status of people with epilepsy was mostly evaluated by clinicians through their own clinical experience instead of the standardized diagnosis procedure. The face-to-face clinical evaluation of patients was hardly achievable because of the high risk of COVID-19 infection in the hospital. The clinician-based observation would underestimate the prevalence of depression and anxiety among people with epilepsy, which indicates that our survey results may be conservative. Third, the economic status, medical resource, and epilepsy prevalence in different areas were potential confounders in this survey. Compared to other areas, we did not find a significantly lower GDP, number of beds and clinicians, proportion of the urban population, or significantly higher epilepsy prevalence in Hubei. We recognized that there may be other potentially relevant factors that have not been taken into account. Fourth, all the questions in the questionnaire should be designed as open-ended or multi-perspective questions to avoid inductivity. Fifth, the board members of CAAE are likely to be top experts of epilepsy in China. Therefore, our study might have neglected opinions from epileptologists other than CAAE board members. Some of them are working in less developed areas and may have met with different situations in clinical practice. Finally, we only received questionnaires from 3 responders in the highrisk area Hubei Pfrovince. This small sample size made the survey less representative and may cause a selection bias. The non-significant difference of some characteristics, e.g. patients with status epilepticus among three epidemic severity areas might also due to the limited sample size. Further larger sampled studies are needed to verify our results.

\section{Conclusion}

This study illuminated the most common dilemma faced by people with epilepsy in policy circumstances during the COVID-19 epidemic in China. The opinions raised by Chinese epileptologists may provide reference for epilepsy care in other countries. Further studies on the physical and psychological health of people with epilepsy in this public health crisis are needed.

\section{Supplementary information}

Supplementary information accompanies this paper at https://doi.org/10. 1186/s42494-020-00030-0.

Additional file 1: Table A.1. Characteristics of COVID-19 epidemic areas with different severity in 31 provinces.

\section{Abbreviations}

ASMs: Anti-seizure medications; CAAE: China Association Against Epilepsy; COVID-19: Coronavirus disease 2019; GDP: Gross domestic product; WHO: World Health Organization

\section{Acknowledgments}

This survey was conducted on behalf of the China Association Against Epilepsy (CAAE). The authors thank CAAE secretaries Hui Zhang, Lirong Duan, and Xueya Hao for their comments and efforts on questionnaire collection. Names and affiliations of survey responders are listed below:

\begin{tabular}{|c|c|}
\hline Names & Affiliations \\
\hline Lixin Cai & Peking University First Hospital \\
\hline Dezhi Cao & Shenzhen Children's Hospital \\
\hline Chen Chen & The First Affiliated Hospital of Zhengzhou University \\
\hline $\begin{array}{l}\text { Guohong } \\
\text { Chen }\end{array}$ & Henan Children's Hospital \\
\hline Lei Chen & West China Hospital, Sichuan University \\
\hline Qian Chen & The Children's Hospital, Capital Institute of Pediatrics \\
\hline Shengli Chen & Chongqing Three Gorges Central Hospital \\
\hline Shuhua Chen & The Children's Hospital, Capital Institute of Pediatrics \\
\hline Yan Chen & The First Hospital of China Medical University \\
\hline Yanhui Chen & Fujian Medical University Union Hospital \\
\hline $\begin{array}{l}\text { Yangmei } \\
\text { Chen }\end{array}$ & $\begin{array}{l}\text { The Second Affiliated Hospital of Chongqing Medical } \\
\text { University }\end{array}$ \\
\hline Ziyi Chen & The First Affiliated Hospital, Sun Yat-sen University \\
\hline Qilin Dai & The First Affiliated Hospital, Sun Yat-sen University \\
\hline Xuejun Deng & $\begin{array}{l}\text { Union Hospital affiliated to Tongji Medical College of } \\
\text { Huazhong University of Science and Technology }\end{array}$ \\
\hline $\begin{array}{l}\text { Yanchun } \\
\text { Deng }\end{array}$ & Xijing Hospital, Air Force Medical University \\
\hline Jing Ding & Zhongshan Hospital, Fudan University \\
\hline Meiping Ding & $\begin{array}{l}\text { The Second Affiliated Hospital Zhejiang University } \\
\text { School of Medicine }\end{array}$ \\
\hline $\begin{array}{l}\text { Wanchen } \\
\text { Dou }\end{array}$ & Peking Union Medical College Hospital \\
\hline Xiaoqin Fan & Ningbo Medical Center Lihuili Hospital \\
\hline Fang Fang & Beijing Children's Hospital, Capital Medical University \\
\hline
\end{tabular}


(Continued)

\begin{tabular}{ll}
\hline Xianming Fu & Anhui Provincial Hospital \\
Li Gao & Henan Provincial People's Hospital \\
Wei Gao & Peking University Shougang Hospital \\
Zaifen Gao & Qilu Children's Hospital of Shandong University \\
Zhiwei Gao & Affiliated Hospital of Nantong University \\
Yuguang & Sanbo Brain Hospital Capital Medical University \\
Guan & \\
Qiang Guo & Guangdong 999 Brain Hospital \\
Xiaodong & The 988 Hospital of the People's Liberation Army \\
Guo & \\
Xiong Han & Henan Provincial People's Hospital \\
Yanbing Han & First Affiliated Hospital of Kunming Medical University \\
Weicheng & Second Hospital of Shanxi Medical University \\
Hao & \\
Yanqiu Hao & $\begin{array}{l}\text { The Second Affiliated Hospital of Harbin Medical } \\
\text { University }\end{array}$
\end{tabular}

Yujun Hao The First Affiliated Hospital of Xinjiang Medical University

Zhiyi He The First Hospital of China Medical University

Mei Hou Qingdao Women and Children's Hospital

Jie Hu Huashan Hospital, Fudan University

Shaoping The Second Affiliated Hospital of Xi'an Jiaotong

Huang University

Wen Jiang Xijing Hospital, Air Force Medical University

Yuwu Jiang Peking University First Hospital

Li Jiang Children's Hospital of Chongqing Medical University

Liri Jin Peking Union Medical College Hospital

Baomin Li Qilu Hospital of Shandong University

Hongyan Li Xinjiang People's Hospital

Ling Li Xinhua Hospital Affiliated to Shanghai Jiao Tong University School of Medicine

Qifu Li The First Affiliated Hospital of Hainan Medical College

Yunlin Li The Children's Hospital, Capital Institute of Pediatrics

Yajun Lian The First Affiliated Hospital of Zhengzhou University

Jianmin Liang The First Hospital of Jilin University

Shuli Liang Beijing Children's Hospital, Capital Medical University

Weihong Lin The First Hospital of Jilin University

Zhiguo Lin The First Affiliated Hospital of Harbin Medical University

Ling Liu

Shiyong Liu

West China Hospital, Sichuan University

Songyan Liu China-Japan Union Hospital of Jilin University

Xiaoming Liu Xuzhou Children's Hospital

Xiaorong Liu The Second Affiliated Hospital of Guangzhou Medical University

Xuewu Liu Qilu Hospital of Shandong University

Yonghong Xijing Hospital, Air Force Medical University

Liu

Zhisheng Liu Wuhan Women and Children's Health Care Center

Lili Long Xiangya Hospital Central South University
(Continued)

Guoming Sanbo Brain Hospital Capital Medical University

Luan

Rong Luo West China Second University Hospital, Sichuan University

Xinming Luo The Second Affiliated Hospital of Nanchang University

Sha Ma The First People's Hospital of Yunnan Province

Hongmei The First Hospital of Jilin University

Meng

Zhengping First Hospital of Shanxi Medical University

Niu

Jing Peng Xiangya Hospital Central South University

Xiaolan Peng The First People's Hospital of Lanzhou City

Ruobing Qian Anhui Provincial Hospital

Liankun Ren Xuanwu Hospital Capital Medical University

Yiyan Ruan The maternal \& Children Health Hospital of Guangxi Zhuang Autonomous Region

Xiaomei Shu Affiliated Hospital of Zunyi Medical University

Lisen Sui Guangdong Province Traditional Chinese Medical Hospital

Meizhen Sun First Hospital of Shanxi Medical University

Wei Sun Xuanwu Hospital Capital Medical University

Yan Sun Xinjiang People's Hospital

Jinyong Tian Guizhou Provincial Peoples Hospital

Xin Wang Zhongshan Hospital, Fudan University

Furong Wang Tongji Hospital affiliated to Tongji Medical College of Huazhong University of Science and Technology

Guofu Wang The First People's Hospital of Foshan City

Huayan The First Affiliated Hospital of Fujian Medical University

Wang

Qun Wang Beijing Tiantan Hospital, Capital Medical University

Shuang The Second Affiliated Hospital Zhejiang University

Wang School of Medicine

Tiancheng Lanzhou University Second Hospital

Wang

Weiwei Wang Peking University First Hospital

Xiaofei Wang Beijing Children's Hospital, Capital Medical University

Xinjun Wang The Fifth Affiliated Hospital of Zhengzhou University

Xiuxia Wang The Second Hospital of Hebei Medical University

Xu Wang Beijing Children's Hospital, Capital Medical University

Xuefeng The First Affiliated Hospital of Chongqing Medical

Wang University

Huaping Wu Jiangxi Provincial Children's Hospital

Yuan Wu The First Affiliated Hospital of Guangxi Medical University

Zhezhi Xia The Children's Hospital, Zhejiang University School of Medicine

Huiqin Xu The First Affiliated Hospital of Wenzhou Medical University

Jianyang Xu Huai'an Third People's Hospital

Zucai Xu Affiliated Hospital of Zunyi Medical University

Xianrui Xu General Hospital of Ningxia Medical University 
(Continued)

\begin{tabular}{|c|c|}
\hline Bin Yang & Anhui Provincial Children's Hospital \\
\hline $\begin{array}{l}\text { Guanglu } \\
\text { Yang }\end{array}$ & $\begin{array}{l}\text { The Affiliated Hospital of Inner Mongolia Medical } \\
\text { University }\end{array}$ \\
\hline Jian Yang & Capital Institute of Pediatrics \\
\hline Jun Yang & General Hospital of Xinjiang Military Region \\
\hline Liming Yang & Hunan Children's Hospital \\
\hline $\begin{array}{l}\text { Pengfan } \\
\text { Yang }\end{array}$ & The 900 Hospital of the People's Liberation Army \\
\hline Yi Yao & Shenzhen Children's Hospital \\
\hline Fei Yin & Xiangya Hospital Central South University \\
\hline Shaoya Yin & Tianjin Huanhu Hospital \\
\hline Yunli Yu & The Affiliated Hospital of Guizhou Medical University \\
\hline Juming Yu & Affiliated Hospital of North Sichuan Medical College \\
\hline Tao Yu & Xuanwu Hospital Capital Medical University \\
\hline Qing Yu & General Hospital of Tianjin Medical University \\
\hline Shikun Zhan & $\begin{array}{l}\text { Ruijin Hospital, Shanghai Jiaotong University School of } \\
\text { Medicine }\end{array}$ \\
\hline Hua Zhang & First Affiliated Hospital of Xi'an Jiaotong University \\
\hline $\begin{array}{l}\text { Jianguo } \\
\text { Zhang }\end{array}$ & Beijing Tiantan Hospital, Capital Medical University \\
\hline Kai Zhang & Beijing Tiantan Hospital, Capital Medical University \\
\hline Ming Zhang & The Second Affiliated Hospital of Nanchang University \\
\hline $\begin{array}{l}\text { Xinding } \\
\text { Zhang }\end{array}$ & Lanzhou University Second Hospital \\
\hline $\begin{array}{l}\text { Yuehua } \\
\text { Zhang }\end{array}$ & Peking University First Hospital \\
\hline Yuqin Zhang & Tianjin Children's Hospital \\
\hline Xiuhe Zhao & Qilu Hospital of Shandong University \\
\hline Yuhua Zhao & Tibet Autonomous Region People's Hospital \\
\hline Jinou Zheng & The First Affiliated Hospital of Guangxi Medical University \\
\hline Dong Zhou & West China Hospital, Sichuan University \\
\hline Jian Zhou & Sanbo Brain Hospital Capital Medical University \\
\hline Liemin Zhou & The Seventh Affiliated Hospital, Sun Yat-sen University \\
\hline Nong Zhou & The First Affiliated Hospital of Anhui Medical University \\
\hline $\begin{array}{l}\text { Shuizhen } \\
\text { Zhou }\end{array}$ & Children's Hospital of Fudan University \\
\hline $\begin{array}{l}\text { Wenjing } \\
\text { Zhou }\end{array}$ & Yuquan Hospital of Tsinghua University \\
\hline $\begin{array}{l}\text { Yuanfeng } \\
\text { Zhou }\end{array}$ & Children's Hospital of Fudan University \\
\hline Yulan Zhu & $\begin{array}{l}\text { The Second Affiliated Hospital of Harbin Medical } \\
\text { University }\end{array}$ \\
\hline Liping Zou & The First Medical Center of Chinese PLA General Hospital \\
\hline
\end{tabular}

\section{Authors' contributions}

$\mathrm{SL}$ and $\mathrm{ZH}$ developed the original idea and designed the questionnaire. The China Association Against Epilepsy collected data. ZX and DD analyzed data; $Z X$ and DD wrote the original draft; $S L$ and $Z H$ reviewed and edited the manuscript. The author(s) read and approved the final manuscript.

\section{Funding}

None.

\section{Availability of data and materials}

The datasets used and/or analyzed during the current study are available from the corresponding author on reasonable request.

\section{Ethics approval and consent to participate}

Not applicable.

\section{Consent for publication}

All the authors are consent for the publication of this article.

\section{Competing interests}

The authors declare that they have no competing interests.

Received: 22 September 2020 Accepted: 14 October 2020

Published online: 27 October 2020

\section{References}

1. Lu L, Xiong W, Liu D, Liu J, Yang D, Li N, et al. New onset acute symptomatic seizure and risk factors in coronavirus disease 2019: A retrospective multicenter study. Epilepsia. 2020;61:e49-e53.

2. French JA, Brodie MJ, Caraballo R, Devinsky O, Ding D, Jehi L, et al. Keeping people with epilepsy safe during the Covid-19 pandemic neurology. Neurology. 2020;94:1032-7.

3. Assenza G, Lanzone J, Brigo F, Coppola A, Di Gennaro G, Di Lazzaro V, et al. Epilepsy care in the time of COVID-19 pandemic in Italy: risk factors for seizure worsening. Front Neurol. 2020;11:737.

4. Alkhotani A, Siddiqui Ml, Almuntashri F, Baothman R. The effect of COVID-19 pandemic on seizure control and self-reported stress on patient with epilepsy. Epilepsy Behav. 2020;112:107323.

5. Hao X, Zhou D, Li Z, Zeng G, Hao N, Li E, et al. Severe psychological distress among epilepsy patients during the COVID-19 outbreak in Southwest China. Epilepsia. 2020;61:1166-73.

6. Chinese Center For Disease Control And Prevention. Epidemic dynamic on 2020 Jan 13. Available at: http://www.chinacdc.cn/jkzt/crb/zl/szkb_11803/ jszl 11809/202001/t20200119_211278.html. Accessed 8 June 2020.

7. Chinese Center For Disease Control And Prevention. Epidemic dynamic on 2020 Mar 31. Available at: http://www.chinacdc.cn/jkzt/crb/zl/szkb_11803/ jszl_11809/202004/t20200401_215729.html. Accessed 8 June 2020.

\section{Ready to submit your research? Choose BMC and benefit from:}

- fast, convenient online submission

- thorough peer review by experienced researchers in your field

- rapid publication on acceptance

- support for research data, including large and complex data types

- gold Open Access which fosters wider collaboration and increased citations

- maximum visibility for your research: over $100 \mathrm{M}$ website views per year

At BMC, research is always in progress.

Learn more biomedcentral.com/submissions 